OCU-PHYS 440

February, 2016

\title{
$q$-Vertex Operator from 5D Nekrasov Function
}

\author{
H. Itoyama $a^{a, 6 冈, ~ T . ~ O o t a ~}$ \\ ${ }^{a}$ Department of Mathematics and Physics, Graduate School of Science \\ Osaka City University \\ ${ }^{b}$ Osaka City University Advanced Mathematical Institute (OCAMI) \\ 3-3-138, Sugimoto, Sumiyoshi-ku, Osaka, 558-8585, Japan
}

\begin{abstract}
The five dimensional AGT correspondence implies the connection between the $q$-deformed Virasoro block and the $5 \mathrm{~d}$ Nekrasov partition function. In this paper, we determine a $q$-deformation of the four-point block in the Coulomb gas representation from the 5d Nekrasov function, and obtain an expression of the $q$-deformed vertex operator. If we use only one kind of the $q$-vertex operators, one of the insertion points of them must be modified in order to hold the $2 \mathrm{~d} / 5 \mathrm{~d}$ correspondence.
\end{abstract}

*e-mail: itoyama@sci.osaka-cu.ac.jp

†e-mail: toota@sci.osaka-cu.ac.jp

‡e-mail: yoshioka@sci.osaka-cu.ac.jp 


\section{Introduction}

Twenty years ago, a $q$-deformation of the Virasoro algebra ( $q$-Virasoro algebra) was introduced in [1, 2, 3]1. It is closely related to the one dimensional XYZ Heisenberg chain model or to a two-dimensional solvable lattice model (the Andrews-Baxter-Forrester model). One motivation for the $q$-deformation is to study thermodynamic limit of these models using the representation theory of this deformed algebra.

In ordinary minimal conformal field theories (CFT), the singular vectors have connection with the Jack symmetric functions indexed by a rectangular partition [6]. There is a generalization of the Jack functions, called Macdonald symmetric functions [7]. The guiding principle for deformation in [3] is such that the singular vectors of the deformed algebra are expressed in terms of the Macdonald functions. The defining relation of the $q$-Virasoro algebra and the screening currents are well established in [3].

On the other hand, the representations of the $q$-Virasoro algebra is still not well understood. In particular, we have no proper definition of $q$-deformed primary fields (vertex operators). The vertex operators or intertwining operators of the $q$-Virasoro algebra for the minimal cases are considered in [8, 9, 10]. These $q$-vertex operators have "good" commutation relations with the $q$-Virasoro generators. But general criterion for goodness is not known.

Recently, the $q$-Virasoro algebra has collected renewed interests due to the $q$-deformed/lifted version (or K-theoretic five dimensional version) of the (W)AGT conjecture. The (W)AGT relation [11, 12] implies the two dimensional correlation functions (conformal blocks) of the Virasoro/W algebra are identical to the four dimensional Nekrasov partition functions. For this $2 \mathrm{~d} / 4 \mathrm{~d}$ correspondence, see for example [13, 14, 15, 16, 17, 18, 19, 20, 21, 22, 23, 24, 25, 26, 27, 28, 29, 30, 31, 32]. The deformed/lifted (W)AGT suggests that the $q$-deformed Virasoro/W blocks in two dimensional theories are identified with the Nekrasov partition function of the five dimensional gauge theories [33, 34]. See also [35, 36, 37, 38, 39, 40, 41, 42, 43] for the 5d AGT. More general 2d/6d correspondence is also discussed in [37, 44, 45, 46].

Assuming the $q$-deformed/lifted version of (W)AGT conjecture, one can fix the form of the $q$ vertex operator. The conformal blocks have a Coulomb gas representation (the Dotsenko-Fateev integral representation). A simple recipe for the $q$-deformation of the conformal block in the Coulomb gas representation is proposed in [35] without constructing the $q$-operators. We consider the problem of operator realization of the deformed block in order to determine the $q$-vertex operator.

In [19], it is shown that the Dotsenko-Fateev representation of the four-point conformal block is related to multiple integrals with the Selberg measure. The Kadell's formula gives the average of the Jack polynomials with respect to this measure. Using the formula, we compared the conformal block with the 4d Nekrasov function and found agreement.

In this paper, we consider a straightforward $q$-deformation of [19]. Kaneko obtained a $q$ deformed version of the Kadell's formula [47]. This formula gives the average of the Macdonald polynomials with respect to the $q$-deformed Selberg measure. By adopting it as a calculational tool, and starting from the $5 \mathrm{~d} S U(2)$ Nekrasov function, we obtained the $q$-deformed (Jackson) integral representation of the four-point block. Using a free field representation of the $q$-screening charges, we have determined an explicit form of the $q$-vertex operator. This is one of our main results.

\footnotetext{
${ }^{1} \mathrm{~A} q$-deformation of $W$ algebra of type $A_{n}$ is treated in [4, 5].
} 
If we use only one kind of the $q$-vertex operators, it turns out that one of the insertion points of them must be modified in order to match the $q$-block with the Nekrasov function. We have no simple explanation on this modification.

This paper is organized as follows. In the next section, after reviewing the $q$-Virasoro algebra and its screening charges, we give explicitly the vertex operator for the $q$-Virasoro algebra such that the $2 \mathrm{~d} / 5 \mathrm{~d}$ correspondence is established. The $q$-deformed version of the Coulomb gas representation of the four-point block is constructed. In Section 3, a brief review of the 5 d Nekrasov partition function is given. In Section 4 , we perform the $\Lambda_{0}$-expansion of the $q$-Virasoro block. The parameter dictionary of $2 \mathrm{~d} / 5 \mathrm{~d}$ correspondence is presented. We display the explicit form of the first order term in $\Lambda_{0}$ expansion. In Section 5 , we carry out the comparison with the $5 \mathrm{~d}$ Nekrasov partition function. In this process, we obtain non-trivial relations among some quantities. In Appendix A, the two-point correlation function for the $q$-Virasoro vertex operator and the $q$-screening current are given. In Appendix B, the $q$-Selberg integral and Kaneko's formula are presented.

\section{2 q-deformed conformal block}

In this section, we first explain our convention for the $q$-deformed Virasoro algebra and its screening charges. Then, we propose a $q$-deformed vertex operator. We also introduce a "modified" fourpoint block $\mathcal{B}\left(\Lambda_{0}\right)$.

\section{$2.1 \quad q$-Virasoro algebra}

Let us consider a $q$-Heisenberg algebra with generators

$$
\alpha_{n}(n \in \mathbb{Z}), \quad Q
$$

satisfying the following defining relations:

$$
\begin{gathered}
{\left[\alpha_{n}, \alpha_{m}\right]=-\frac{1}{n} \frac{\left(1-q^{n}\right)\left(1-t^{-n}\right)}{\left(1+p^{n}\right)} \delta_{n+m, 0}, \quad(n \neq 0),} \\
{\left[\alpha_{n}, Q\right]=\delta_{n, 0} .}
\end{gathered}
$$

Here $p=q / t$. The $q$-deformed Virasoro algebra can be realized by this algebra [3]

$$
\mathcal{T}(z)=: \exp \left(\sum_{n \neq 0} \alpha_{n} z^{-n}\right): p^{1 / 2} q^{\sqrt{\beta} \alpha_{0}}+: \exp \left(-\sum_{n \neq 0} \alpha_{n}(p z)^{-n}\right): p^{-1 / 2} q^{-\sqrt{\beta} \alpha_{0}} .
$$

Here $\beta$ is defined by the relation $t=q^{\beta}$.

This operator satisfies the defining relation of the $q$-Virasoro algebra:

$$
f\left(z^{\prime} / z\right) \mathcal{T}(z) \mathcal{T}\left(z^{\prime}\right)-f\left(z / z^{\prime}\right) \mathcal{T}\left(z^{\prime}\right) \mathcal{T}(z)=\frac{(1-q)\left(1-t^{-1}\right)}{(1-p)}\left[\delta\left(p z / z^{\prime}\right)-\delta\left(p^{-1} z / z^{\prime}\right)\right]
$$

where

$$
f(z)=\exp \left(\sum_{n=1}^{\infty} \frac{1}{n} \frac{\left(1-q^{n}\right)\left(1-t^{-n}\right)}{\left(1+p^{n}\right)} z^{n}\right), \quad \delta(z)=\sum_{n \in \mathbb{Z}} z^{n} .
$$

In the following part, we assume that $|q|<1$. 


\subsection{Screening charges}

A screening current for the $q$-Virasoro algebra is defined by

$$
S_{+}(z)=: \mathrm{e}^{\tilde{\varphi}^{(+)}(z)}:
$$

where

$$
\tilde{\varphi}^{(+)}(z)=\sqrt{\beta} Q+2 \sqrt{\beta} \alpha_{0} \log z+\sum_{n \neq 0} \frac{1+p^{-n}}{1-q^{n}} \alpha_{n} z^{-n} .
$$

This screening current $S_{+}$commutes with the $q$-Virasoro generators up to a total $q$-derivative:

$$
\left[T(z), S_{+}\left(z^{\prime}\right)\right]=-(1-q)\left(1-t^{-1}\right) \frac{\mathrm{d}_{q}}{\mathrm{~d}_{q} z^{\prime}}\left[\delta\left(z^{\prime} / z\right) p^{-1 / 2} z^{\prime} A_{+}\left(z^{\prime}\right)\right],
$$

where

$$
A_{+}(z)=: \exp \left(\sum_{n \neq 0} \frac{\left(1+t^{n}\right)}{\left(1-q^{n}\right)} \alpha_{n} z^{-n}\right): \mathrm{e}^{\sqrt{\beta} Q} q^{-\sqrt{\beta} \alpha_{0}} z^{2 \sqrt{\beta} \alpha_{0}} .
$$

The $q$-derivative is given by

$$
\frac{\mathrm{d}_{q} f(z)}{\mathrm{d}_{q} z}=\frac{f(z)-f(q z)}{z-q z} .
$$

There is another screening current $S_{-}(z)$ which commutes with the $q$-Virasoro generators up to a total $t$-derivative. But we will not use $S_{-}(z)$ in this paper, hence we do not introduce it.

Using two different "integration ranges", two screening charges $Q_{+}, Q_{+}^{\prime}$ are defined by

$$
Q_{+}:=\int_{0}^{\Lambda_{0}} \mathrm{~d}_{q} z S_{+}(z), \quad Q_{+}^{\prime}:=\int_{1}^{\infty} \mathrm{d}_{q} z S_{+}(z) \equiv \int_{0}^{1} \frac{\mathrm{d}_{q} y}{y^{2}} S_{+}(1 / y) .
$$

Here we use the Jackson integral

$$
\int_{0}^{a} \mathrm{~d}_{q} z f(z)=(1-q) \sum_{n=0}^{\infty} f\left(a q^{n}\right) a q^{n} .
$$

\subsection{Vertex operators for the $q$-Virasoro algebra}

Vertex operators for the $q$-Virasoro algebra are considered in [8, 9, 10, 34]. In [8, 9, 10], a $q$ deformation of primary operators $V_{r, s}(z)=: \mathrm{e}^{(1 / 2) \alpha_{r, s} \phi(z)}$ : in the minimal CFT model is introduced. Here $\alpha_{r, s}=(1-r) \sqrt{\beta}-(1-s) / \sqrt{\beta}$ with rational $\beta$. The normalization of chiral boson is chosen as $\left\langle\phi(z) \phi\left(z^{\prime}\right)\right\rangle=2 \log \left(z-z^{\prime}\right)$. But we are not interested in these types of $q$-vertex operators.

The $q$-vertex operator determined from the $5 \mathrm{~d}$ Nekrasov function is the following. For a complex parameter $u$, let us define a vertex operator of the $q$-deformed Virasoro algebra by

$$
V_{u}(z):=: \mathrm{e}^{\Phi_{u}(z)}:
$$

where

$$
\Phi_{u}(z):=\frac{u}{\sqrt{\beta}}\left(\frac{1}{2} Q+\alpha_{0} \log z\right)+\sum_{n \neq 0} \frac{\left(q^{n u}-1\right)}{\left(1-q^{n}\right)\left(1-t^{-n}\right)} \alpha_{n} z^{-n} .
$$


This is essentially equivalent to the vertex operator $V_{U}^{1}(z)$ in $[34]^{2}$ with $U=q^{-u}$.

In the $q \rightarrow 1$ limit with keeping $\beta=\log t / \log q$ fixed, this vertex operator becomes a free field representation of the Virasoro primary operator with scaling dimension $\Delta=u(u-2 \beta+2) /(4 \beta)$.

\section{$2.4 q$-deformed Coulomb gas representation}

A $q$-deformed version of the Coulomb gas representation of the four-point block is defined by

$$
\left\langle V_{u_{1}}(0) V_{u_{2}}\left(\Lambda_{0}\right) V_{u_{3}}(1) V_{u_{4}}(\infty)\left(Q_{+}\right)^{N_{+}}\left(Q_{+}^{\prime}\right)^{N_{-}}\right\rangle .
$$

But we will consider the following "modified" four-point block:

$$
\mathcal{B}\left(\Lambda_{0}\right)=\left\langle V_{u_{1}}(0) V_{u_{2}}\left(\Lambda_{0}\right) V_{u_{3}}\left(q^{u_{3}+1}\right) V_{u_{4}}(\infty)\left(Q_{+}\right)^{N_{+}}\left(Q_{+}^{\prime}\right)^{N_{-}}\right\rangle .
$$

The four vertex operators are inserted at $z_{1}=0, z_{2}=\Lambda_{0}, z_{3}=q^{u_{3}+1}$ and $z_{4}=\infty$. Note that $z_{3}=q^{u_{3}+1}$, instead of $z_{3}=1$. We have no simple explanation on the position of the third vertex operator. With this choice of $z_{3}$, the modified four-point conformal block (2.17) coincides with the five dimensional Nekrasov partition function of the $S U(2)$ gauge theory with $N_{f}=4$.

In (2.17), the parameters $u_{i}$ should obey the "momentum conservation condition":

$$
u_{1}+u_{2}+u_{3}+u_{4}+2 \beta\left(N_{+}+N_{-}\right)+2(1-\beta)=0 \text {. }
$$

The parameters $u_{i}$ used here is related to the parameters $\alpha_{i}$ in [19] as $u_{i}=\sqrt{\beta} \alpha_{i}$.

For simplicity, we assume that

$$
0<\left|\Lambda_{0}\right|<\left|q^{u_{3}+1}\right|<1 .
$$

Using the two-point correlators in Appendix A, we have

$$
\begin{aligned}
\mathcal{B}\left(\Lambda_{0}\right) & =\mathcal{V}_{0}\left(\Lambda_{0}\right)\left(N_{+}\right) !\left(N_{-}\right) ! \int_{\mathcal{C}_{N_{+}}\left(\left[0, \Lambda_{0}\right]\right)} \mathrm{d}_{q}^{N_{+}} z \int_{\mathcal{C}_{N_{-}}^{\prime}([1, \infty])} \mathrm{d}_{q}^{N_{-}} z^{\prime} \\
& \times \prod_{i=1}^{N_{+}} z_{i}^{u_{1}} \frac{\left(q^{-u_{3}} z_{i} ; q\right)_{\infty}\left(q z_{i} / \Lambda_{0} ; q\right)_{\infty}}{\left(z_{i} ; q\right)_{\infty}\left(q^{u_{2}+1} z_{i} / \Lambda_{0} ; q\right)_{\infty}} \prod_{j=1}^{N_{-}}\left(z_{j}^{\prime}\right)^{u_{1}+u_{2}+u_{3}} \frac{\left(q / z_{j}^{\prime} ; q\right)_{\infty}\left(q^{-u_{2}} \Lambda_{0} / z_{j}^{\prime} ; q\right)_{\infty}}{\left(q^{u_{3}+1} / z_{j}^{\prime} ; q\right)_{\infty}\left(\Lambda_{0} / z_{j}^{\prime} ; q\right)_{\infty}} \\
& \times \prod_{1 \leq i<j \leq N_{+}} z_{i}^{2 \beta}\left(1-\frac{z_{j}}{z_{i}}\right) \frac{\left(q^{1-\beta} z_{j} / z_{i} ; q\right)_{\infty}}{\left(q^{\beta} z_{j} / z_{i} ; q\right)_{\infty}} \prod_{1 \leq i<j \leq N_{-}}\left(z_{j}^{\prime}\right)^{2 \beta}\left(1-\frac{z_{i}^{\prime}}{z_{j}^{\prime}}\right) \frac{\left(q^{1-\beta} z_{i}^{\prime} / z_{j}^{\prime} ; q\right)_{\infty}}{\left(q^{\beta} z_{i}^{\prime} / z_{j}^{\prime} ; q\right)_{\infty}} \\
& \times \prod_{i=1}^{N_{+}} \prod_{j=1}^{N_{-}}\left(z_{j}^{\prime}\right)^{2 \beta}\left(1-\frac{z_{i}}{z_{j}^{\prime}}\right) \frac{\left(q^{1-\beta} z_{i} / z_{j}^{\prime} ; q\right)_{\infty}}{\left(q^{\beta} z_{i} / z_{j}^{\prime} ; q\right)_{\infty}} .
\end{aligned}
$$

Here the constant $\mathcal{V}_{0}\left(\Lambda_{0}\right)$ is given by

$$
\begin{aligned}
\mathcal{V}_{0}\left(\Lambda_{0}\right):= & \Lambda_{0}^{1 /(2 \beta) u_{1} u_{2}+u_{2} N_{+}} q^{\left(u_{1}+u_{2}+2 \beta N_{+}\right) u_{3}\left(u_{3}+1\right) /(2 \beta)} \\
& \times \exp \left(-\sum_{n=1}^{\infty} \frac{1}{n} \frac{\left(q^{-n u_{3}}-1\right)\left(q^{-n u_{2}}-1\right)}{\left(1-q^{n}\right)\left(1-t^{n}\right)\left(1+p^{n}\right)} \Lambda_{0}^{n}\right) .
\end{aligned}
$$

${ }^{2}$ By identification of the fundamental bosons $h_{n}^{1}$ in $\left[34\right.$ with our $\alpha_{n}$, we have $V_{q^{-u}}^{1}(z)=V_{u}\left(q^{u / 2} z\right) q^{u^{2} \alpha_{0} /(2 \sqrt{\beta})}$. 
The "range" $\mathcal{C}_{N_{+}}\left(\left[0, \Lambda_{0}\right]\right)$ of the Jackson integral for $\left\{z_{i}\right\}_{i=1,2, \ldots, N_{+}}$are the interval $\left[0, \Lambda_{0}\right]$ with the additional condition

$$
0 \leq\left|z_{N_{+}}\right|<\left|z_{N_{+}-1}\right|<\cdots<\left|z_{2}\right|<\left|z_{1}\right| \leq\left|\Lambda_{0}\right|
$$

Recall that we have assumed $|q|<1$. Hence, this condition means that, for a parameterization $z_{i}=\Lambda_{0} q^{k_{i}}$ in the Jackson integral, the non-negative integers $k_{i}$ are summed over with

$$
0 \leq k_{1}<k_{2}<\cdots<k_{N_{+}}
$$

Similarly, the "range" $\mathcal{C}_{N_{-}}^{\prime}([1, \infty])$ is chosen such that

$$
1 \leq\left|z_{1}^{\prime}\right|<\left|z_{2}^{\prime}\right|<\cdots<\left|z_{N_{-}}^{\prime}\right|<\infty
$$

It means that $z_{j}^{\prime}=q^{-k_{j}^{\prime}}$ with $0 \leq k_{1}^{\prime}<k_{2}^{\prime}<\cdots<k_{N_{-}}^{\prime}$.

By the following coordinate transformation:

$$
z_{i}=\Lambda_{0} x_{i},\left(i=1,2, \ldots, N_{+}\right), \quad z_{j}^{\prime}=1 / y_{j},\left(j=1,2, \ldots, N_{-}\right),
$$

we have

$$
\begin{aligned}
\mathcal{B}\left(\Lambda_{0}\right) & =\mathcal{V}_{0}^{\prime}\left(\Lambda_{0}\right)\left(N_{+}\right) !\left(N_{-}\right) ! \int_{C_{N_{+}}([0,1])} \mathrm{d}_{q}^{N_{+}} x \int_{C_{N_{-}}([0,1])} \mathrm{d}_{q}^{N_{-}} y \\
& \times \prod_{i=1}^{N_{+}} x_{i}^{u_{1}} \frac{\left(q x_{i} ; q\right)_{\infty}}{\left(q^{u_{2}+1} x_{i} ; q\right)_{\infty}} \prod_{1 \leq i<j \leq N_{+}} x_{i}^{2 \beta}\left(1-\frac{x_{j}}{x_{i}}\right) \frac{\left(q^{1-\beta} x_{j} / x_{i} ; q\right)_{\infty}}{\left(q^{\beta} x_{j} / x_{i} ; q\right)_{\infty}} \\
& \times \prod_{j=1}^{N_{-}} y_{j}^{u_{4}} \frac{\left(q y_{j} ; q\right)_{\infty}}{\left(q^{u_{3}+1} y_{j} ; q\right)_{\infty}} \prod_{1 \leq i<j \leq N_{-}} y_{i}^{2 \beta}\left(1-\frac{y_{j}}{y_{i}}\right) \frac{\left(q^{1-\beta} y_{j} / y_{i} ; q\right)_{\infty}}{\left(q^{\beta} y_{j} / y_{i} ; q\right)_{\infty}} \\
& \times \prod_{i=1}^{N_{+}} \frac{\left(q^{-u_{3}} \Lambda_{0} x_{i} ; q\right)_{\infty}}{\left(\Lambda_{0} x_{i} ; q\right)_{\infty}} \prod_{j=1}^{N_{-}} \frac{\left(q^{-u_{2}} \Lambda_{0} y_{j} ; q\right)_{\infty}}{\left(\Lambda_{0} y_{j} ; q\right)_{\infty}} \prod_{i=1}^{N_{+}} \prod_{j=1}^{N_{-}}\left(1-\Lambda_{0} x_{i} y_{j}\right) \frac{\left(q^{1-\beta} \Lambda_{0} x_{i} y_{j} ; q\right)_{\infty}}{\left(q^{\beta} \Lambda_{0} x_{i} y_{j} ; q\right)_{\infty}},
\end{aligned}
$$

where

$$
\begin{aligned}
\mathcal{V}_{0}^{\prime}\left(\Lambda_{0}\right)= & \mathcal{V}_{0}\left(\Lambda_{0}\right) \Lambda_{0}^{N_{+}\left(u_{1}+1\right)+\beta N_{+}\left(N_{+}-1\right)} \\
= & \Lambda_{0}^{1 /(2 \beta) u_{1} u_{2}+N_{+}\left(u_{1}+u_{2}+1\right)+\beta N_{+}\left(N_{+}-1\right)} q^{\left(u_{1}+u_{2}+2 \beta N_{+}\right) u_{3}\left(u_{3}+1\right) /(2 \beta)} \\
& \quad \times \exp \left(-\sum_{n=1}^{\infty} \frac{1}{n} \frac{\left(q^{-n u_{3}}-1\right)\left(q^{-n u_{2}}-1\right)}{\left(1-q^{n}\right)\left(1-t^{n}\right)\left(1+p^{n}\right)} \Lambda_{0}^{n}\right) .
\end{aligned}
$$

The Jackson integrals in $(2.26)$ are taken for the range $[0,1]$ under the following conditions

$$
\begin{aligned}
& 0 \leq\left|x_{N_{+}}\right|<\left|x_{N_{+}-1}\right|<\cdots<\left|x_{2}\right|<\left|x_{1}\right| \leq 1 \\
& 0 \leq\left|y_{N_{-}}\right|<\left|y_{N_{-}-1}\right|<\cdots<\left|y_{2}\right|<\left|y_{1}\right| \leq 1
\end{aligned}
$$

Let

$$
\mathcal{B}_{0}\left(\Lambda_{0}\right):=\mathcal{B}\left(\Lambda_{0}\right) / \mathcal{V}_{0}^{\prime}\left(\Lambda_{0}\right)
$$


At $\Lambda_{0}=0, \mathcal{B}_{0}\left(\Lambda_{0}\right)$ factorizes into a product of two $q$-deformed Selberg integrals:

$$
\mathcal{B}_{0}(0)=S_{N_{+}}^{(q)}\left(u_{1}, u_{2}, \beta\right) S_{N_{-}}^{(q)}\left(u_{4}, u_{3}, \beta\right)
$$

where

$$
\begin{aligned}
& S_{N}^{(q)}\left(u_{1}, u_{2}, \beta\right) \\
& =N ! \int_{C_{N}([0,1])} \mathrm{d}_{q}^{N} z \prod_{i=1}^{N} z_{i}^{u_{1}} \frac{\left(q z_{i} ; q\right)_{\infty}}{\left(q^{u_{2}+1} z_{i} ; q\right)_{\infty}} \prod_{1 \leq i<j \leq N} z_{i}^{2 \beta}\left(1-\frac{z_{j}}{z_{i}}\right) \frac{\left(q^{1-\beta} z_{j} / z_{i} ; q\right)_{\infty}}{\left(q^{\beta} z_{j} / z_{i} ; q\right)_{\infty}} \\
& =N !(1-q)^{N} \sum_{0 \leq k_{1}<k_{2}<\cdots<k_{N}} \prod_{i=1}^{N} q^{\left(u_{1}+1\right) k_{i}} \frac{\left(q^{k_{i}+1} ; q\right)_{\infty}}{\left(q^{k_{i}+u_{2}+1} ; q\right)_{\infty}} \prod_{1 \leq i<j \leq N} q^{2 \beta k_{i}}\left(1-q^{k_{j}-k_{i}}\right) \frac{\left(q^{k_{j}-k_{i}+1-\beta} ; q\right)_{\infty}}{\left(q^{k_{j}-k_{i}+\beta} ; q\right)_{\infty}} .
\end{aligned}
$$

When $\beta$ is a positive integer, (2.32) becomes

$$
\begin{aligned}
& S_{N}^{(q)}\left(u_{1}, u_{2}, \beta\right) \\
& =\int_{[0,1]^{N}} \mathrm{~d}_{q}^{N} z \prod_{i=1}^{N} z_{i}^{u_{1}} \frac{\left(q z_{i} ; q\right)_{\infty}}{\left(q^{u_{2}+1} z_{i} ; q\right)_{\infty}} \prod_{1 \leq i<j \leq N}\left\{\left(z_{i}-z_{j}\right) \prod_{\ell=0}^{2 \beta-2}\left(z_{i}-q^{\ell+1-\beta} z_{j}\right)\right\} .
\end{aligned}
$$

This integral (2.33) is evaluated as

$$
S_{N}^{(q)}\left(u_{1}, u_{2}, \beta\right)=N ! q^{A_{N}\left(u_{1}, \beta\right)} \prod_{j=1}^{N} \frac{\Gamma_{q}\left(u_{1}+1+(N-j) \beta\right) \Gamma_{q}\left(u_{2}+1+(N-j) \beta\right) \Gamma_{q}(j \beta)}{\Gamma_{q}\left(u_{1}+u_{2}+2+(2 N-j-1) \beta\right) \Gamma_{q}(\beta)},
$$

where $\Gamma_{q}(x)$ is the $q$-Gamma function and

$$
A_{N}\left(u_{1}, \beta\right)=\frac{1}{2} N(N-1)\left(u_{1}+1\right) \beta+\frac{1}{3} N(N-1)(N-2) \beta^{2}, \quad(\beta \text { : a positive integer }) .
$$

We expect that (2.34) also holds when $\beta$ is not a positive integer with a modification of $A_{N}\left(u_{1}, \beta\right)$ from (2.35).

For example, for positive $u_{1}$ and $u_{2}$, the small $q$ behavior of $S_{N}^{(q)}$ is given by $N ! q^{A_{N}}+\cdots$. When $0<\beta<2$, the leading contribution in the sum (2.32) comes from the term with $k_{i}=(i-1)$. Hence, it seems that in this case,

$$
A_{N}\left(u_{1}, \beta\right)=\frac{1}{2} N(N-1)\left(u_{1}+1\right)+\frac{1}{3} N(N-1)(N-2) \beta, \quad(0<\beta<2) .
$$

\subsection{Remark: $q \rightarrow 1$ limit}

In this subsection, we comment on the $q \rightarrow 1$ limit of various objects in previous subsections.

For notational simplicity, let us introduce the following functions:

$$
D_{N}^{(q)}\left(u_{1}, u_{2}, \beta ; z\right)=\prod_{j=1}^{N} z_{j}^{u_{1}} \frac{\left(q z_{j} ; q\right)_{\infty}}{\left(q^{u_{2}+1} z_{j} ; q\right)_{\infty}} \prod_{1 \leq i<j \leq N} z_{i}^{2 \beta-1} \frac{\left(q^{1-\beta} z_{j} / z_{i} ; q\right)_{\infty}}{\left(q^{\beta} z_{j} / z_{i} ; q\right)_{\infty}}\left(z_{i}-z_{j}\right)
$$




$$
\begin{aligned}
& F_{N_{+}, N_{-}}^{(q)}\left(u_{2}, u_{3}, \beta, \Lambda_{0} ; x, y\right) \\
& =\prod_{i=1}^{N_{+}} \frac{\left(\Lambda_{0} q^{-u_{3}} x_{i} ; q\right)_{\infty}}{\left(\Lambda_{0} x_{i} ; q\right)_{\infty}} \prod_{j=1}^{N_{-}} \frac{\left(\Lambda_{0} q^{-u_{2}} y_{j} ; q\right)_{\infty}}{\left(\Lambda_{0} y_{j} ; q\right)_{\infty}} \prod_{i=1}^{N_{+}} \prod_{j=1}^{N_{-}} \frac{\left(q^{1-\beta} \Lambda_{0} x_{i} y_{j} ; q\right)_{\infty}}{\left(q^{\beta} \Lambda_{0} x_{i} y_{j} ; q\right)_{\infty}}\left(1-\Lambda_{0} x_{i} y_{j}\right) .
\end{aligned}
$$

In the $q \rightarrow 1$ limit, these objects behave as follows:

$$
\begin{aligned}
& \lim _{q \rightarrow 1} D_{N}^{(q)}\left(u_{1}, u_{2}, \beta ; z\right)=\prod_{j=1}^{N} z_{j}^{u_{1}}\left(1-z_{j}\right)^{u_{2}} \prod_{1 \leq i<j \leq N}\left(z_{i}-z_{j}\right)^{2 \beta} . \\
& \lim _{q \rightarrow 1} F_{N_{+}, N_{-}}^{(q)}\left(u_{2}, u_{3}, \beta, \Lambda_{0} ; x, y\right) \\
& =\prod_{i=1}^{N_{+}}\left(1-\Lambda_{0} x_{i}\right)^{u_{3}} \prod_{j=1}^{N_{-}}\left(1-\Lambda_{0} y_{j}\right)^{u_{2}} \prod_{i=1}^{N_{+}} \prod_{j=1}^{N_{-}}\left(1-\Lambda_{0} x_{i} y_{j}\right)^{2 \beta}
\end{aligned}
$$

Hence, (2.26) goes to Eq.(2.8) of [19].

Using (2.37), the $q$-deformed Selberg integral (2.32) can be written as

$$
S_{N}^{(q)}\left(u_{1}, u_{2}, \beta\right)=N ! \int_{C_{N}([0,1])} \mathrm{d}_{q}^{N} z D_{N}^{(q)}\left(u_{1}, u_{2}, \beta ; z\right) .
$$

In the $q \rightarrow 1$ limit, the $q$-Selberg integral (2.32) with (2.34) goes to the ordinary Selberg integral:

$$
\begin{aligned}
\lim _{q \rightarrow 1} S_{N}^{(q)}\left(u_{1}, u_{2}, \beta\right) & =\int_{0}^{1} \mathrm{~d}^{N} z \prod_{j=1}^{N} z_{j}^{u_{1}}\left(1-z_{j}\right)^{u_{2}} \prod_{1 \leq i<j \leq N}\left|z_{i}-z_{j}\right|^{2 \beta} \\
& =\prod_{j=1}^{N} \frac{\Gamma\left(u_{1}+1+(N-j) \beta\right) \Gamma\left(u_{2}+1+(N-j) \beta\right) \Gamma(1+j \beta)}{\Gamma\left(u_{1}+u_{2}+2+(2 N-j-1) \beta\right) \Gamma(1+\beta)} .
\end{aligned}
$$

Here we have used $\lim _{q \rightarrow 1} \Gamma_{q}(x)=\Gamma(x)$ and

$$
N ! \prod_{j=1}^{N} \frac{\Gamma(j \beta)}{\Gamma(\beta)}=\prod_{j=1}^{N} \frac{\Gamma(1+j \beta)}{\Gamma(1+\beta)} .
$$

The $q \rightarrow 1$ limit is related to the $2 \mathrm{~d}$ CFT and the $4 \mathrm{~d}$ gauge theory on the flat space. While root of unity limits of $q$ are related to $2 \mathrm{~d}$ supersymmetric/parafermionic theories and the $4 \mathrm{~d}$ gauge theories on ALE spaces [48, 49, 50, 51, 52, 53, 54, 55, 56, 57, 58, 55, 60].

\section{5d Nekrasov partition function}

In this section, we briefly review the Nekrasov partition function on $\mathbb{R}^{3,1} \times S^{1}$. We denote the radius of $S^{1}$ by $R$. The five dimensional $S U(N)$ Nekrasov partition function with $N_{f}=2 N$ fundamental matters can be found in [61, 62] (see also [63]). We follow the notation of [34] and consider the $N=2$ case. 
The instanton part of the five dimensional $S U(2)$ Nekrasov partition function with $N_{f}=4$ fundamental matters is given by

$$
Z_{2}^{\text {inst }}(Q ; \Lambda)=\sum_{\lambda, \mu} Z_{\lambda, \mu}^{( \pm)}\left(\frac{\Lambda_{\alpha}^{ \pm}}{v^{2}}\right)^{|\lambda|+|\mu|}
$$

where

$$
Z_{\lambda, \mu}^{( \pm)}=\frac{\prod_{i=1}^{2} N_{\lambda,(0)}\left(v Q_{1} / Q_{i}^{ \pm}\right) N_{\mu,(0)}\left(v Q_{2} / Q_{i}^{ \pm}\right) N_{(0), \lambda}\left(v Q_{i}^{\mp} / Q_{1}\right) N_{(0), \mu}\left(v Q_{i}^{\mp} / Q_{2}\right)}{N_{\lambda, \lambda}(1) N_{\mu, \mu}(1) N_{\lambda, \mu}\left(Q_{1} / Q_{2}\right) N_{\mu, \lambda}\left(Q_{2} / Q_{1}\right)} .
$$

Here $v=(q / t)^{1 / 2}=p^{1 / 2}$. The summation in (3.1) is over a pair of partitions $(\lambda, \mu)$. The function $N_{\lambda \mu}(Q)$ is defined by

$$
\begin{aligned}
N_{\lambda \mu}(Q) & :=\prod_{(i, j) \in \lambda}\left(1-Q q^{\lambda_{i}-j} t^{\mu_{j}^{\prime}-i+1}\right) \prod_{(i, j) \in \mu}\left(1-Q q^{-\mu_{i}+j-1} t^{-\lambda_{j}^{\prime}+i}\right) \\
& =\prod_{(i, j) \in \mu}\left(1-Q q^{\lambda_{i}-j} t^{\mu_{j}^{\prime}-i+1}\right) \prod_{(i, j) \in \lambda}\left(1-Q q^{-\mu_{i}+j-1} t^{-\lambda_{j}^{\prime}+i}\right) .
\end{aligned}
$$

Here $\lambda^{\prime}$ is the conjugate partition of $\lambda$.

The parameters in (3.1) are $q, t, \Lambda, Q_{1}, Q_{2}, Q_{1}^{ \pm}$and $Q_{2}^{ \pm} . q$ and $t$ are related to the $\Omega$-background parameters $\epsilon_{1}$ and $\epsilon_{2}$ :

$$
q=\mathrm{e}^{R \epsilon_{2}}, \quad t=\mathrm{e}^{-R \epsilon_{1}}
$$

The parameters $Q_{1}$ and $Q_{2}$ are related to the vev of the adjoint scalar in the 4 d theory:

$$
v Q_{1}=\mathrm{e}^{R a}, \quad v Q_{2}=\mathrm{e}^{-R a}
$$

while $Q_{i}^{ \pm}$are related to the mass of fundamental matters:

$$
Q_{1}^{+}=\mathrm{e}^{-R m_{1}}, \quad Q_{2}^{+}=\mathrm{e}^{-R m_{2}}, \quad Q_{1}^{-}=\mathrm{e}^{-R m_{3}}, \quad Q_{2}^{-}=\mathrm{e}^{-R m_{4}} .
$$

The expansion parameters $\Lambda_{\alpha}^{ \pm}$are defined by

$$
\Lambda_{\alpha}^{ \pm}=\Lambda^{4}\left(\frac{Q_{1}^{ \pm} Q_{2}^{ \pm}}{Q_{1}^{\mp} Q_{2}^{\mp}}\right)^{1 / 2}=\Lambda^{4} \mathrm{e}^{ \pm(1 / 2) R\left(m_{3}+m_{4}-m_{1}-m_{2}\right)} .
$$

Hence the relation between $Z_{\lambda, \mu}^{( \pm)}$is given by

$$
Z_{\lambda, \mu}^{(+)}=\mathrm{e}^{(|\lambda|+|\mu|) R\left(m_{1}+m_{2}-m_{3}-m_{4}\right)} Z_{\lambda, \mu}^{(-)} .
$$

\subsection{First order: $Z_{(1),(0)}^{( \pm)}$and $Z_{(0),(1)}^{( \pm)}$}

Using (3.4), (3.5) and (3.6), $Z_{\lambda, \mu}^{ \pm}$with $|\lambda|+|\mu|=1$ can be written as

$$
Z_{(1),(0)}^{(+)}=\frac{\left(1-\mathrm{e}^{R\left(a+m_{1}\right)}\right)\left(1-\mathrm{e}^{R\left(a+m_{2}\right)}\right)\left(1-\mathrm{e}^{-R\left(a+m_{3}\right)}\right)\left(1-\mathrm{e}^{-R\left(a+m_{4}\right)}\right)}{\left(1-\mathrm{e}^{-R \epsilon_{1}}\right)\left(1-\mathrm{e}^{-R \epsilon_{2}}\right)\left(1-\mathrm{e}^{2 R a}\right)\left(1-\mathrm{e}^{-R(2 a+\epsilon)}\right)},
$$




$$
\begin{aligned}
& Z_{(0),(1)}^{(+)}=\frac{\left(1-\mathrm{e}^{-R\left(a-m_{1}\right)}\right)\left(1-\mathrm{e}^{-R\left(a-m_{2}\right)}\right)\left(1-\mathrm{e}^{R\left(a-m_{3}\right)}\right)\left(1-\mathrm{e}^{R\left(a-m_{4}\right)}\right)}{\left(1-\mathrm{e}^{-R \epsilon_{1}}\right)\left(1-\mathrm{e}^{-R \epsilon_{2}}\right)\left(1-\mathrm{e}^{-2 R a}\right)\left(1-\mathrm{e}^{R(2 a-\epsilon)}\right)} . \\
& Z_{(1),(0)}^{(-)}=\frac{\left(1-\mathrm{e}^{-R\left(a+m_{1}\right)}\right)\left(1-\mathrm{e}^{-R\left(a+m_{2}\right)}\right)\left(1-\mathrm{e}^{R\left(a+m_{3}\right)}\right)\left(1-\mathrm{e}^{R\left(a+m_{4}\right)}\right)}{\left(1-\mathrm{e}^{-R \epsilon_{1}}\right)\left(1-\mathrm{e}^{-R \epsilon_{2}}\right)\left(1-\mathrm{e}^{2 R a}\right)\left(1-\mathrm{e}^{-R(2 a+\epsilon)}\right)}, \\
& Z_{(0),(1)}^{(-)}=\frac{\left(1-\mathrm{e}^{R\left(a-m_{1}\right)}\right)\left(1-\mathrm{e}^{R\left(a-m_{2}\right)}\right)\left(1-\mathrm{e}^{-R\left(a-m_{3}\right)}\right)\left(1-\mathrm{e}^{-R\left(a-m_{4}\right)}\right)}{\left(1-\mathrm{e}^{-R \epsilon_{1}}\right)\left(1-\mathrm{e}^{-R \epsilon_{2}}\right)\left(1-\mathrm{e}^{-2 R a}\right)\left(1-\mathrm{e}^{R(2 a-\epsilon)}\right)} .
\end{aligned}
$$

Here $\epsilon=\epsilon_{1}+\epsilon_{2}$.

In the $R \rightarrow 0$ limit, these terms reproduce the $4 \mathrm{~d}$ results:

$$
\begin{aligned}
& \lim _{R \rightarrow 0} Z_{(1),(0)}^{( \pm)}=\frac{\left(a+m_{1}\right)\left(a+m_{2}\right)\left(a+m_{3}\right)\left(a+m_{4}\right)}{2 a(2 a+\epsilon) g_{s}^{2}} \\
& \lim _{R \rightarrow 0} Z_{(0),(1)}^{( \pm)}=\frac{\left(a-m_{1}\right)\left(a-m_{2}\right)\left(a-m_{3}\right)\left(a-m_{4}\right)}{2 a(2 a-\epsilon) g_{s}^{2}} .
\end{aligned}
$$

\section{$4 \quad \Lambda_{0}$ expansion of $q$-block}

In this section, we study the expansion of the modified blocks in the $\Lambda_{0}$ parameter.

\subsection{Method of calculation in $q$-block}

In order to compare the $q$-block (2.30) with the $5 \mathrm{~d}$ Nekrasov function (3.1), let us introduce the following function

$$
\mathcal{A}\left(\Lambda_{0}\right):=\frac{\mathcal{B}_{0}\left(\Lambda_{0}\right)}{\mathcal{B}_{0}(0)}=\left\langle\left\langle F_{N_{+}, N_{-}}^{(q)}\left(u_{2}, u_{3}, \beta, \Lambda_{0} ; x, y\right)\right\rangle\right\rangle=1+\sum_{n=1}^{\infty} \Lambda_{0}^{n} \mathcal{A}_{n} .
$$

Here

$$
\langle\langle f(x, y)\rangle\rangle=\left\langle\langle f(x, y)\rangle_{+}\right\rangle_{-},
$$

and \langle\rangle$_{ \pm}$is the average with respect to the $q$-deformed Selberg measure:

$$
\begin{aligned}
& \langle f(x)\rangle_{+}=\frac{1}{S_{N_{+}}^{(q)}\left(u_{1}, u_{2}, \beta\right)} \int_{C_{N_{+}}([0,1])} \mathrm{d}_{q}^{N_{+}} x D_{N_{+}}^{(q)}\left(u_{1}, u_{2}, \beta ; x\right) f(x), \\
& \langle f(y)\rangle_{-}=\frac{1}{S_{N_{-}}^{(q)}\left(u_{4}, u_{3}, \beta\right)} \int_{C_{N_{-}}([0,1])} \mathrm{d}_{q}^{N_{-}} y D_{N_{-}}^{(q)}\left(u_{4}, u_{3}, \beta ; y\right) f(y) .
\end{aligned}
$$

Note that

$$
\begin{array}{r}
F_{N_{+}, N_{-}}^{(q)}=\exp \left[-\sum_{k=1}^{\infty} \frac{\Lambda_{0}^{k}}{k} \frac{\left(1-t^{k}\right)}{\left(1-q^{k}\right)}\left\{\left(p_{k}(x)-\frac{1-q^{-u_{2} k}}{1-t^{k}}\right) p_{k}(y)\right.\right. \\
\left.\left.+p_{k}(x)\left((q / t)^{k} p_{k}(y)-\frac{1-q^{-u_{3} k}}{1-t^{k}}\right)\right\}\right],
\end{array}
$$


where $p_{k}$ denotes the power sum:

$$
p_{k}(x)=\sum_{i=1}^{N_{+}} x_{i}^{k}, \quad p_{k}(y)=\sum_{j=1}^{N_{-}} y_{j}^{k} .
$$

We conjecture that the Kaneko's formula (B.8) also holds for the contour $C_{N_{ \pm}}([0,1])$. We have checked it for small values of $N$ and $\beta$.

Then, we can calculate $\mathcal{A}_{n}$ by using the average of the Macdonald polynomial $P_{\lambda}$ :

$$
\begin{aligned}
\left\langle P_{\lambda}(x ; q, t)\right\rangle_{+} & =\frac{\left(t^{N_{+}}\right)_{\lambda}^{(q, t)}\left(q^{u_{1}+1} t^{\left(N_{+}-1\right)}\right)_{\lambda}^{(q, t)}}{h_{\lambda}(q, t)\left(q^{u_{1}+u_{2}+2} t^{2\left(N_{+}-1\right)}\right)_{\lambda}^{(q, t)}} \\
& =\prod_{(i, j) \in \lambda} \frac{\left(t^{i-1}-q^{j-1} t^{N_{+}}\right)\left(t^{i-1}-q^{u_{1}+j} t^{N_{+}-1}\right)}{\left(1-q^{\lambda_{i}-j} t^{\lambda_{j}^{\prime}-i+1}\right)\left(t^{i-1}-q^{u_{1}+u_{2}+j+1} t^{2 N_{+}-2}\right)} \\
\left\langle P_{\lambda}(y ; q, t)\right\rangle_{-}= & \frac{\left(t^{N_{-}}\right)_{\lambda}^{(q, t)}\left(q^{u_{4}+1} t^{\left(N_{-}-1\right)}\right)_{\lambda}^{(q, t)}}{h_{\lambda}(q, t)\left(q^{u_{3}+u_{4}+2} t^{2\left(N_{-}-1\right)}\right)_{\lambda}^{(q, t)}} \\
& =\prod_{(i, j) \in \lambda} \frac{\left(t^{i-1}-q^{j-1} t^{N_{-}}\right)\left(t^{i-1}-q^{u_{4}+j} t^{N_{-}-1}\right)}{\left(1-q^{\lambda_{i}-j} t^{\lambda_{j}^{\prime}-i+1}\right)\left(t^{i-1}-q^{u_{3}+u_{4}+j+1} t^{2 N_{-}-2}\right)}
\end{aligned}
$$

\subsection{Parameter dictionary}

In [19], we have used the following identification of $\beta$ with the $\Omega$-background parameters $\epsilon_{1}$ and $\epsilon_{2}:$

$$
\epsilon_{1}=\sqrt{\beta} g_{s}, \quad \epsilon_{2}=-\frac{1}{\sqrt{\beta}} g_{s}, \quad \epsilon=\epsilon_{1}+\epsilon_{2}=\left(\sqrt{\beta}-\frac{1}{\sqrt{\beta}}\right) g_{s} .
$$

In this choice, it holds that $\epsilon_{1} \epsilon_{2}=-g_{s}^{2}$.

With this identification, (3.4) yields the following relations:

$$
q=\mathrm{e}^{R \epsilon_{2}}=\mathrm{e}^{-R g_{s} / \sqrt{\beta}}, \quad t=q^{\beta}=\mathrm{e}^{-R \epsilon_{1}}=\mathrm{e}^{-R g_{s} \sqrt{\beta}}, \quad v=(q / t)^{1 / 2}=\mathrm{e}^{(1 / 2) R \epsilon} .
$$

The momentum conservation condition (2.18) can be rewritten as

$$
q^{u_{1}+u_{2}+u_{3}+u_{4}+2} t^{2\left(N_{+}+N_{-}\right)-2}=1 .
$$

The $2 \mathrm{~d} / 4 \mathrm{~d}$ dictionary used in [19] now converted to the following $2 \mathrm{~d} / 5 \mathrm{~d}$ dictionary:

$$
\begin{array}{cc}
t^{N_{+}}=\mathrm{e}^{-R\left(a-m_{2}\right)}, & t^{N_{-}}=\mathrm{e}^{R\left(a+m_{3}\right)}, \\
q^{u_{1}}=\mathrm{e}^{-R\left(m_{2}-m_{1}+\epsilon\right)}, & q^{u_{2}}=\mathrm{e}^{-R\left(m_{1}+m_{2}\right)}, \\
q^{u_{3}}=\mathrm{e}^{-R\left(m_{3}+m_{4}\right)}, & q^{u_{4}}=\mathrm{e}^{-R\left(m_{3}-m_{4}+\epsilon\right)} .
\end{array}
$$

Some useful relations are given by

$$
\begin{gathered}
q^{u_{1}+1} t^{-1}=\mathrm{e}^{-R\left(m_{2}-m_{1}\right)}, \quad q^{u_{2}}=\mathrm{e}^{-R\left(m_{1}+m_{2}\right)}, \\
q^{u_{3}}=\mathrm{e}^{-R\left(m_{3}+m_{4}\right)}, \quad q^{u_{4}+1} t^{-1}=\mathrm{e}^{-R\left(m_{3}-m_{4}\right)}, \\
q^{u_{1}+u_{2}+1} t^{2 N_{+}-1}=\mathrm{e}^{-2 R a}, \quad q^{u_{3}+u_{4}+1} t^{2 N_{-}-1}=\mathrm{e}^{2 R a} .
\end{gathered}
$$




\subsection{First order: $\mathcal{A}_{1}$}

The first order term in the $\Lambda_{0}$-expansion (4.1) is given by

$$
\begin{aligned}
\mathcal{A}_{1} & =-\frac{(1-t)}{(1-q)}\left(1+v^{2}\right)\left\langle p_{1}(x)\right\rangle_{+}\left\langle p_{1}(y)\right\rangle_{-} \\
& +\frac{1-q^{-u_{3}}}{1-q}\left\langle p_{1}(x)\right\rangle_{+}+\frac{1-q^{-u_{2}}}{1-q}\left\langle p_{1}(y)\right\rangle_{-} .
\end{aligned}
$$

Note that the following identity holds for any $A$ and $v$ :

$$
\frac{1-v^{2} A}{1-A}+\frac{1-v^{2} A^{-1}}{1-A^{-1}}=1+v^{2}
$$

Specializing this relation by setting $A$ to

$$
A=q^{u_{1}+u_{2}+1} t^{2 N_{+}-1}, \quad A^{-1}=q^{u_{3}+u_{4}+1} t^{2 N_{-}-1},
$$

we have

$$
1+v^{2}=\frac{1-q^{u_{1}+u_{2}+2} t^{2 N_{+}-2}}{1-q^{u_{1}+u_{2}+1} t^{2 N_{+}-1}}+\frac{1-q^{u_{3}+u_{4}+2} t^{2 N_{-}-2}}{1-q^{u_{3}+u_{4}+1} t^{2 N_{-}-1}} .
$$

With help of (4.21), a non-trivial decomposition of $\mathcal{A}_{1}$ is obtained:

$$
\mathcal{A}_{1}=\mathcal{A}_{(1),(0)}+\mathcal{A}_{(0),(1)},
$$

where

$$
\begin{aligned}
& \mathcal{A}_{(1),(0)}=\left\{-\frac{(1-t)\left(1-q^{u_{1}+u_{2}+2} t^{2 N_{+}-2}\right)}{(1-q)\left(1-q^{u_{1}+u_{2}+1} t^{2 N_{+}-1}\right)}\left\langle p_{1}(x)\right\rangle_{+}+\frac{\left(1-q^{-u_{2}}\right)}{(1-q)}\right\}\left\langle p_{1}(y)\right\rangle_{-}, \\
& \mathcal{A}_{(0),(1)}=\left\langle p_{1}(x)\right\rangle_{+}\left\{-\frac{(1-t)\left(1-q^{u_{3}+u_{4}+2} t^{2 N_{-}-2}\right)}{(1-q)\left(1-q^{u_{3}+u_{4}+1} t^{2 N_{-}-1}\right)}\left\langle p_{1}(y)\right\rangle_{-}+\frac{\left(1-q^{-u_{3}}\right)}{(1-q)}\right\} .
\end{aligned}
$$

The averages for the Macdonald polynomial with $\lambda=(1)$ are given by

$$
\begin{aligned}
& \left\langle P_{(1)}(x ; q, t)\right\rangle_{+}=\left\langle p_{1}(x)\right\rangle_{+}=\frac{\left(1-t^{N_{+}}\right)\left(1-q^{u_{1}+1} t^{N_{+}-1}\right)}{(1-t)\left(1-q^{u_{1}+u_{2}+2} t^{2 N_{+}-2}\right)}, \\
& \left\langle P_{(1)}(y ; q, t)\right\rangle_{-}=\left\langle p_{1}(y)\right\rangle_{-}=\frac{\left(1-t^{N_{-}}\right)\left(1-q^{u_{4}+1} t^{N_{-}-1}\right)}{(1-t)\left(1-q^{u_{3}+u_{4}+2} t^{2 N_{-}-2}\right)} .
\end{aligned}
$$

By substituting these expressions into factors in curly bracket of (4.23) and (4.24), we can see that

$$
\begin{aligned}
& \left\{-\frac{(1-t)\left(1-q^{u_{1}+u_{2}+2} t^{2 N_{+}-2}\right)}{(1-q)\left(1-q^{u_{1}+u_{2}+1} t^{2 N_{+}-1}\right)}\left\langle p_{1}(x)\right\rangle_{+}+\frac{\left(1-q^{-u_{2}}\right)}{(1-q)}\right\} \\
& =-\frac{\left(1-t^{N_{+}}\right)\left(1-q^{u_{1}+1} t^{N_{+}-1}\right)}{(1-q)\left(1-q^{u_{1}+u_{2}+1} t^{2 N_{+}-1}\right)}+\frac{\left(1-q^{-u_{2}}\right)}{(1-q)} \\
& =-q^{u_{1}+u_{2}+1} t^{2 N_{+}-1} \frac{\left(1-q^{-u_{2}} t^{-N_{+}}\right)\left(1-q^{-u_{1}-u_{2}-1} t^{1-N_{+}}\right)}{(1-q)\left(1-q^{u_{1}+u_{2}+1} t^{2 N_{+}-1}\right)}
\end{aligned}
$$




$$
\begin{aligned}
& \left\{-\frac{(1-t)\left(1-q^{u_{3}+u_{4}+2} t^{2 N_{-}-2}\right)}{(1-q)\left(1-q^{u_{3}+u_{4}+1} t^{2 N_{-}-1}\right)}\left\langle p_{1}(y)\right\rangle_{-}+\frac{\left(1-q^{-u_{3}}\right)}{(1-q)}\right\} \\
& =-\frac{\left(1-t^{N_{-}}\right)\left(1-q^{u_{4}+1} t^{N_{-}-1}\right)}{(1-q)\left(1-q^{u_{3}+u_{4}+1} t^{2 N_{-}-1}\right)}+\frac{\left(1-q^{-u_{3}}\right)}{(1-q)} \\
& =-q^{u_{3}+u_{4}+1} t^{2 N_{-}-1} \frac{\left(1-q^{-u_{3}} t^{-N_{-}}\right)\left(1-q^{-u_{3}-u_{4}-1} t^{1-N_{-}}\right)}{(1-q)\left(1-q^{u_{3}+u_{4}+1} t^{2 N_{-}-1}\right)} .
\end{aligned}
$$

Consequently, we have explicit form of $\mathcal{A}_{(1),(0)}$ and $\mathcal{A}_{(0),(1)}$ :

$$
\begin{aligned}
& \mathcal{A}_{(1),(0)} \\
& =-q^{u_{1}+u_{2}+1} t^{2 N_{+}-1} \frac{\left(1-q^{-u_{2}} t^{-N_{+}}\right)\left(1-q^{-u_{1}-u_{2}-1} t^{1-N_{+}}\right)\left(1-t^{N_{-}}\right)\left(1-q^{u_{4}+1} t^{N_{-}-1}\right)}{(1-q)(1-t)\left(1-q^{u_{1}+u_{2}+1} t^{2 N_{+}-1}\right)\left(1-q^{u_{3}+u_{4}+2} t^{2 N_{-}-2}\right)}, \\
& \mathcal{A}_{(0),(1)} \\
& =-q^{u_{3}+u_{4}+1} t^{2 N_{-}-1} \frac{\left(1-q^{-u_{3}} t^{-N_{-}}\right)\left(1-q^{-u_{3}-u_{4}-1} t^{1-N_{-}}\right)\left(1-t^{N_{+}}\right)\left(1-q^{u_{1}+1} t^{N_{+}-1}\right)}{(1-q)(1-t)\left(1-q^{u_{3}+u_{4}+1} t^{2 N_{-}-1}\right)\left(1-q^{u_{1}+u_{2}+2} t^{2 N_{+}-2}\right)} .
\end{aligned}
$$

\subsubsection{In terms of parameters of gauge theory}

Let us rewrite the parameters in (4.29) and (4.30) by the gauge theory parameters.

Using

$$
\begin{gathered}
q^{-u_{2}}=\mathrm{e}^{R\left(m_{1}+m_{2}\right)}, \quad q^{-u_{3}}=\mathrm{e}^{R\left(m_{3}+m_{4}\right)} . \\
t^{N_{+}}=\mathrm{e}^{-R\left(a-m_{2}\right)}, \quad q^{u_{1}+1} t^{N_{+}-1}=\mathrm{e}^{-R\left(a-m_{1}\right)}, \\
q^{u_{1}+u_{2}+2} t^{2 N_{+}-2}=\mathrm{e}^{-R(2 a-\epsilon)}, \\
t^{N_{-}}=\mathrm{e}^{R\left(a+m_{3}\right)}, \quad q^{u_{4}+1} t^{N_{-}-1}=\mathrm{e}^{R\left(a+m_{4}\right)},
\end{gathered}
$$

etc., we have

$$
\begin{gathered}
\left\langle p_{1}(x)\right\rangle_{+}=\frac{\left(1-\mathrm{e}^{-R\left(a-m_{1}\right)}\right)\left(1-\mathrm{e}^{-R\left(a-m_{2}\right)}\right)}{\left(1-\mathrm{e}^{-R \epsilon_{1}}\right)\left(1-\mathrm{e}^{-R(2 a-\epsilon)}\right)}, \\
\left\langle p_{1}(y)\right\rangle_{-}=\frac{\left(1-\mathrm{e}^{R\left(a+m_{3}\right)}\right)\left(1-\mathrm{e}^{R\left(a+m_{4}\right)}\right)}{\left(1-\mathrm{e}^{-R \epsilon_{1}}\right)\left(1-\mathrm{e}^{R(2 a+\epsilon)}\right)}, \\
-\frac{(1-t)\left(1-q^{u_{1}+u_{2}+2} t^{2 N_{+}-2}\right)}{(1-q)\left(1-q^{u_{1}+u_{2}+1} t^{2 N_{+}-1}\right)}\left\langle p_{1}(x)\right\rangle_{+}+\frac{\left(1-q^{-u_{2}}\right)}{(1-q)}=\frac{\left(1-\mathrm{e}^{R\left(a+m_{1}\right)}\right)\left(1-\mathrm{e}^{R\left(a+m_{2}\right)}\right)}{\left(1-\mathrm{e}^{R \epsilon_{2}}\right)\left(1-\mathrm{e}^{2 R a}\right)}, \\
-\frac{(1-t)\left(1-q^{u_{3}+u_{4}+2} t^{2 N_{-}-2}\right)}{(1-q)\left(1-q^{u_{3}+u_{4}+1} t^{2 N_{-}-1}\right)}\left\langle p_{1}(y)\right\rangle_{-}+\frac{\left(1-q^{-u_{3}}\right)}{(1-q)}=\frac{\left(1-\mathrm{e}^{-R\left(a-m_{3}\right)}\right)\left(1-\mathrm{e}^{-R\left(a-m_{4}\right)}\right)}{\left(1-\mathrm{e}^{R \epsilon_{2}}\right)\left(1-\mathrm{e}^{-2 R a}\right)} .
\end{gathered}
$$

Thus, we finally have the following expressions:

$$
\begin{gathered}
\mathcal{A}_{(1),(0)}=\frac{\left(1-\mathrm{e}^{R\left(a+m_{1}\right)}\right)\left(1-\mathrm{e}^{R\left(a+m_{2}\right)}\right)\left(1-\mathrm{e}^{R\left(a+m_{3}\right)}\right)\left(1-\mathrm{e}^{R\left(a+m_{4}\right)}\right)}{\left(1-\mathrm{e}^{R \epsilon_{2}}\right)\left(1-\mathrm{e}^{-R \epsilon_{1}}\right)\left(1-\mathrm{e}^{2 R a}\right)\left(1-\mathrm{e}^{R(2 a+\epsilon)}\right)}, \\
\mathcal{A}_{(0),(1)}=\frac{\left(1-\mathrm{e}^{-R\left(a-m_{1}\right)}\right)\left(1-\mathrm{e}^{-R\left(a-m_{2}\right)}\right)\left(1-\mathrm{e}^{-R\left(a-m_{3}\right)}\right)\left(1-\mathrm{e}^{-R\left(a-m_{4}\right)}\right)}{\left(1-\mathrm{e}^{R \epsilon_{2}}\right)\left(1-\mathrm{e}^{-R \epsilon_{1}}\right)\left(1-\mathrm{e}^{-2 R a}\right)\left(1-\mathrm{e}^{-R(2 a-\epsilon)}\right)} .
\end{gathered}
$$


Remark: the following identity plays the crucial role in (4.36):

$$
\begin{aligned}
& \mathrm{e}^{-2 R a}\left(1-\mathrm{e}^{R\left(a-m_{1}\right)}\right)\left(1-\mathrm{e}^{R\left(a-m_{2}\right)}\right)+\left(1-\mathrm{e}^{-2 R a}\right)\left(1-\mathrm{e}^{-R\left(m_{1}+m_{2}\right)}\right) \\
& =\left(1-\mathrm{e}^{-R\left(a+m_{1}\right)}\right)\left(1-\mathrm{e}^{-R\left(a+m_{2}\right)}\right),
\end{aligned}
$$

and similarly the following identity is used in (4.37):

$$
\begin{aligned}
& \mathrm{e}^{-2 R a}\left(1-\mathrm{e}^{R\left(a+m_{3}\right)}\right)\left(1-\mathrm{e}^{R\left(a+m_{4}\right)}\right)+\left(1-\mathrm{e}^{-2 R a}\right)\left(1-\mathrm{e}^{R\left(m_{3}+m_{4}\right)}\right) \\
& =\left(1-\mathrm{e}^{-R\left(a-m_{3}\right)}\right)\left(1-\mathrm{e}^{-R\left(a-m_{4}\right)}\right) .
\end{aligned}
$$

\section{Comparison with 5d Nekrasov partition function}

In this section, we compare the modified $q$-block with the Nekrasov function.

We assume that the $2 \mathrm{~d} / 5 \mathrm{~d}$ correspondence holds, i.e.,

$$
\mathcal{A}\left(\Lambda_{0}\right)=Z_{2}^{\text {inst }}(Q ; \Lambda) \text {. }
$$

By comparing (4.38) with (3.9) or (3.11), and (4.39) with (3.10) or (3.12), we can check that

$$
\begin{aligned}
& \mathcal{A}_{(1),(0)}=\left(\frac{t}{q^{2}}\right) \mathrm{e}^{R\left(m_{3}+m_{4}\right)} Z_{(1),(0)}^{(+)}=\left(\frac{t}{q^{2}}\right) \mathrm{e}^{R\left(m_{1}+m_{2}\right)} Z_{(1),(0)}^{(-)}, \\
& \mathcal{A}_{(0),(1)}=\left(\frac{t}{q^{2}}\right) \mathrm{e}^{R\left(m_{3}+m_{4}\right)} Z_{(0),(1)}^{(+)}=\left(\frac{t}{q^{2}}\right) \mathrm{e}^{R\left(m_{1}+m_{2}\right)} Z_{(0),(1)}^{(-)} .
\end{aligned}
$$

Then we must have

$$
\begin{aligned}
& \Lambda_{0} \mathcal{A}_{(1),(0)}=\frac{\Lambda_{\alpha}^{+}}{v^{2}} Z_{(1),(0)}^{(+)}=\frac{\Lambda_{\alpha}^{-}}{v^{2}} Z_{(1),(0)}^{(-)}, \\
& \Lambda_{0} \mathcal{A}_{(0),(1)}=\frac{\Lambda_{\alpha}^{+}}{v^{2}} Z_{(0),(1)}^{(+)}=\frac{\Lambda_{\alpha}^{-}}{v^{2}} Z_{(0),(1)}^{(-)} .
\end{aligned}
$$

The relations (5.2) and (5.3) lead to the following identification of the expansion parameters:

$$
\Lambda_{0}=q \mathrm{e}^{-R\left(m_{3}+m_{4}\right)} \Lambda_{\alpha}^{+}=q \mathrm{e}^{-R\left(m_{1}+m_{2}\right)} \Lambda_{\alpha}^{-}=q \mathrm{e}^{-(1 / 2) R\left(m_{1}+m_{2}+m_{3}+m_{4}\right)} \Lambda^{4} .
$$

This connection is also stated as follows:

$$
\Lambda_{0}=q^{(1 / 2)\left(u_{2}+u_{3}\right)} \Lambda^{4}
$$

Using this identification, (5.1) decomposes into the following identities:

$$
\mathcal{A}_{k}=\left(\frac{t}{q^{2}}\right)^{k} \mathrm{e}^{k R\left(m_{3}+m_{4}\right)} \sum_{|\lambda|+|\mu|=k} Z_{\lambda, \mu}^{(+)}=\left(\frac{t}{q^{2}}\right)^{k} \mathrm{e}^{k R\left(m_{1}+m_{2}\right)} \sum_{|\lambda|+|\mu|=k} Z_{\lambda, \mu}^{(-)} .
$$

We have checked these identities up to $k=4$. These are quite non-trivial relations even for the cases of low order $k$. We expect that these hold for all $k$. Therefore, this gives strong evidence of the $2 \mathrm{~d} / 5 \mathrm{~d}$ correspondence (5.11). 


\section{Acknowledgments}

We would like to thank Mikhail Bershtein and Yusuke Ohkubo for valuable discussions. This work was supported by JSPS KAKENHI Grant Number 15K05059. Support from JSPS/RFBR bilateral collaborations "Faces of matrix models in quantum field theory and statistical mechanics" (H. I. and R. Y.) and "Exploration of Quantum Geometry via Symmetry and Duality" (T. O.) is gratefully appreciated.

\section{A Two-point correlation functions}

In this section, we collect two-point functions utilized in Subsection 2.4.

The (radial ordered) two-point correlation functions for the $q$-deformed vertex operators (2.14) and the screening current (2.7) are given by

$$
\begin{aligned}
& \left\langle V_{u_{1}}\left(z_{1}\right) V_{u_{2}}\left(z_{2}\right)\right\rangle \\
& = \begin{cases}z_{1}^{u_{1} u_{2} /(2 \beta)} \exp \left(-\sum_{n=1}^{\infty} \frac{1}{n} \frac{\left(q^{n u_{1}}-1\right)\left(q^{-n u_{2}}-1\right)}{\left(1-q^{-n}\right)\left(1-t^{n}\right)\left(1+p^{n}\right)}\left(\frac{z_{2}}{z_{1}}\right)^{n}\right), & \left|z_{1}\right|>\left|z_{2}\right|, \\
z_{2}^{u_{1} u_{2} /(2 \beta)} \exp \left(-\sum_{n=1}^{\infty} \frac{1}{n} \frac{\left(q^{n u_{2}}-1\right)\left(q^{-n u_{1}}-1\right)}{\left(1-q^{-n}\right)\left(1-t^{n}\right)\left(1+p^{n}\right)}\left(\frac{z_{1}}{z_{2}}\right)^{n}\right), & \left|z_{2}\right|>\left|z_{1}\right|,\end{cases} \\
& \left\langle V_{u}\left(z_{1}\right) S_{+}\left(z_{2}\right)\right\rangle= \begin{cases}z_{1}^{u} \frac{\left(q z_{2} / z_{1} ; q\right)_{\infty}}{\left(q^{u+1} z_{2} / z_{1} ; q\right)_{\infty}}, & \left|z_{1}\right|>\left|z_{2}\right|, \\
z_{2}^{u} \frac{\left(q^{-u} z_{1} / z_{2} ; q\right)_{\infty}}{\left(z_{1} / z_{2} ; q\right)_{\infty}}, & \left|z_{2}\right|>\left|z_{1}\right|\end{cases} \\
& \left\langle S_{+}\left(z_{1}\right) S_{+}\left(z_{2}\right)\right\rangle= \begin{cases}z_{1}^{2 \beta}\left(1-\frac{z_{2}}{z_{1}}\right) \frac{\left(q^{1-\beta} z_{2} / z_{1} ; q\right)_{\infty}}{\left(q^{\beta} z_{2} / z_{1} ; q\right)_{\infty}}, & \left|z_{1}\right|>\left|z_{2}\right|, \\
z_{2}^{2 \beta}\left(1-\frac{z_{1}}{z_{2}}\right) \frac{\left(q^{1-\beta} z_{1} / z_{2} ; q\right)_{\infty}}{\left(q^{\beta} z_{1} / z_{2} ; q\right)_{\infty}}, & \left|z_{2}\right|>\left|z_{1}\right| .\end{cases}
\end{aligned}
$$

Here

$$
(x ; q)_{\infty}=\prod_{n=0}^{\infty}\left(1-x q^{n}\right)
$$

is the $q$-Pochhammer symbol.

\section{B q-Selberg integral and Kaneko's formula}

In this section, we shortly summarize the $q$-Selberg integral and the Kaneko's formula [47]. 


\section{B.1 $q$-Selberg integral}

Using (2.37), let us consider the following $q$-deformation of the Selberg integral:

$$
S_{N}^{(q)}\left(u_{1}, u_{2}, \beta ; \xi\right):=\int_{[0, \xi \infty]} \mathrm{d}_{q}^{N} z D_{N}^{(q)}\left(u_{1}, u_{2}, \beta ; z\right),
$$

with

$$
\xi=\left(\xi_{1}, \xi_{2}, \ldots, \xi_{N}\right) \in\left(\mathbb{C}^{*}\right)^{N}
$$

Here

$$
\begin{aligned}
& \int_{[0, \xi \infty]} \mathrm{d}_{q}^{N} z f\left(z_{1}, z_{2}, \ldots, z_{N}\right) \\
& =(1-q)^{N}\left(\prod_{j=1}^{N} \xi_{j} \sum_{\left(s_{1}, s_{2}, \ldots, s_{N}\right) \in \mathbb{Z}^{N}} q^{s_{1}+s_{2}+\cdots+s_{N}} f\left(\xi_{1} q^{s_{1}}, \xi_{2} q^{s_{2}}, \ldots, \xi_{N} q^{s_{N}}\right) .\right.
\end{aligned}
$$

For certain value of the parameters $u_{1}, u_{2}, \beta, \xi$ such that the Jackson integral (B.1) converges, the Aomoto's formula [64] implies that (see also [47])

$$
\begin{aligned}
& S_{N}^{(q)}\left(u_{1}, u_{2}, \beta ; \xi\right) \\
& =q^{(1 / 2) N(N-1)^{2} \beta} \prod_{j=1}^{N} \xi_{j}^{u_{1}+2 \beta(N-j)-N+2} \frac{\vartheta\left(\xi_{j} q^{u_{1}+u_{2}+2+(\beta-1)(N-1)}\right) \vartheta\left(q^{u_{2}+1+(j-1) \beta}\right) \vartheta\left(q^{j \beta}\right)}{\vartheta\left(q^{u_{1}+u_{2}+(2 \beta-1)(N-1)-(N-j) \beta}\right) \vartheta\left(\xi_{j} q^{u_{2}}\right) \vartheta\left(q^{\beta}\right)} \\
& \times \prod_{1 \leq i<j \leq N} \frac{\vartheta\left(\xi_{j} / \xi_{i}\right)}{\vartheta\left(q^{\beta} \xi_{j} / \xi_{i}\right)} \prod_{j=1}^{N} \frac{\Gamma_{q}\left(u_{1}+1+(j-1) \beta\right) \Gamma_{q}\left(u_{2}+1+(j-1) \beta\right) \Gamma_{q}(j \beta)}{\Gamma_{q}\left(u_{1}+u_{2}+2+\beta(N+j-2)\right)} .
\end{aligned}
$$

Here $\vartheta(x)$ is the Jacobi elliptic function

$$
\vartheta(x)=(x ; q)_{\infty}(q / x ; q)_{\infty}(q ; q)_{\infty},
$$

and $\Gamma_{q}(x)$ is the $q$-Gamma function

$$
\Gamma_{q}(x)=(1-q)^{1-x} \frac{(q ; q)_{\infty}}{\left(q^{x} ; q\right)_{\infty}} .
$$

\section{B.2 Kaneko's formula}

Let $P_{\lambda}(z ; q, t)$ be the Macdonald polynomial for the variables $z=\left(z_{1}, z_{2}, \ldots, z_{N}\right)$. The average over the Macdonald polynomials is defined by

$$
\left\langle P_{\lambda}(z ; q, t)\right\rangle:=\frac{1}{S_{N}^{(q)}\left(u_{1}, u_{2}, \beta ; \xi\right)} \int_{[0, \xi \infty]} \mathrm{d}_{q}^{N} z P_{\lambda}(z ; q, t) D_{N}^{(q)}\left(u_{1}, u_{2}, \beta ; z\right) .
$$

It is given by [4]

$$
\begin{aligned}
\left\langle P_{\lambda}(z ; q, t)\right\rangle & =\frac{\left(t^{N}\right)_{\lambda}^{(q, t)}\left(q^{u_{1}+1} t^{(N-1)}\right)_{\lambda}^{(q, t)}}{h_{\lambda}(q, t)\left(q^{u_{1}+u_{2}+2} t^{2(N-1)}\right)_{\lambda}^{(q, t)}} \\
& =\prod_{(i, j) \in \lambda} \frac{\left(t^{i-1}-q^{j-1} t^{N_{+}}\right)\left(t^{i-1}-q^{u_{1}+j} t^{N-1}\right)}{\left(1-q^{\lambda_{i}-j} t^{\lambda_{j}^{\prime}-i+1}\right)\left(t^{i-1}-q^{u_{1}+u_{2}+j+1} t^{2 N-2}\right)}
\end{aligned}
$$


Here

$$
\begin{gathered}
(A)_{\lambda}^{(q, t)}=\prod_{s \in \lambda}\left(t^{\ell^{\prime}(s)}-q^{a^{\prime}(s)} A\right)=\prod_{(i, j) \in \lambda}\left(t^{i-1}-q^{j-1} A\right), \\
h_{\lambda}(q, t)=\prod_{s \in \lambda}\left(1-q^{a(s)} t^{\ell(s)+1}\right)=\prod_{(i, j) \in \lambda}\left(1-q^{\lambda^{\prime}-j} t^{\lambda_{j}^{\prime}-i+1}\right) .
\end{gathered}
$$

For a square $s=(i, j)$ in a partition $\lambda$, the arm-length, the leg-length, the arm-colength and the leg-colength are respectively denoted by $a(s), \ell(s), a^{\prime}(s)$ and $\ell^{\prime}(s)$.

Note that the average (B.8) does not depend on the choice of $\xi$.

\section{B.3 Special case}

When $\beta$ is a positive integer, $\beta=k$, by choosing $\xi=\left(1, q^{\beta}, q^{2 \beta}, \cdots, q^{(N-1) \beta}\right)$, the Aomoto's formula (B.4) reduces to the Askey-Habsieger-Kadell's formula [65, 66, 67] :

$$
\begin{aligned}
& \int_{[0,1]^{N}} \mathrm{~d}_{q} z_{1} \wedge \mathrm{d}_{q} z_{2} \wedge \cdots \wedge \mathrm{d}_{q} z_{N} \prod_{i=1}^{N} z_{i}^{u_{1}} \frac{\left(q z_{i} ; q\right)_{\infty}}{\left(q^{u_{2}+1} z_{i} ; q\right)_{\infty}} \prod_{1 \leq i<j \leq N} z_{i}^{2 k} \frac{\left(q^{1-k} z_{j} / z_{i} ; q\right)_{\infty}}{\left(q^{1+k} z_{j} / z_{i} ; q\right)_{\infty}} \\
& =q^{A_{N}} \prod_{i=1}^{N} \frac{\Gamma_{q}\left(u_{1}+1+(N-i) k\right) \Gamma_{q}\left(u_{2}+1+(N-i) k\right) \Gamma_{q}(1+i k)}{\Gamma_{q}\left(u_{1}+u_{2}+2+(2 N-i-1) k\right) \Gamma_{q}(1+k)},
\end{aligned}
$$

where

$$
A_{N}=\frac{1}{2}\left(u_{1}+1\right) k N(N-1)+\frac{1}{3} k^{2} N(N-1)(N-2)
$$

Notice that there is a slight difference between (2.33) and (B.11). In (2.33), the integrand is symmetric under a permutation of $z_{i}$ and $z_{j}$, while in (B.11) it is not the case.

Also, Kadell's formula [67, 47] is obtained as a special case of Kaneko's formula (B.8):

$$
\begin{aligned}
& \int_{[0,1]^{N}} \mathrm{~d}_{q} z_{1} \wedge \mathrm{d}_{q} z_{2} \wedge \cdots \wedge \mathrm{d}_{q} z_{N} P_{\lambda}\left(z ; q, q^{k}\right) \prod_{i=1}^{N} z_{i}^{u_{1}} \frac{\left(q z_{i} ; q\right)_{\infty}}{\left(q^{u_{2}+1} z_{i} ; q\right)_{\infty}} \prod_{1 \leq i<j \leq N} z_{i}^{2 k} \frac{\left(q^{1-k} z_{j} / z_{i} ; q\right)_{\infty}}{\left(q^{1+k} z_{j} / z_{i} ; q\right)_{\infty}} \\
& =q^{A_{N}} P_{\lambda}\left(1, q^{k}, q^{2 k}, \ldots, q^{(N-1) k}\right) \\
& \times \prod_{i=1}^{N} \frac{\Gamma_{q}\left(u_{1}+1+(N-i) k+\lambda_{i}\right) \Gamma_{q}\left(u_{2}+1+(N-i) k\right) \Gamma_{q}(1+i k)}{\Gamma_{q}\left(u_{1}+u_{2}+2+(2 N-i-1) k+\lambda_{i}\right) \Gamma_{q}(1+k)} .
\end{aligned}
$$

Here

$$
P_{\lambda}\left(1, q^{k}, q^{2 k}, \ldots, q^{(N-1) k} ; q, q^{k}\right)=\frac{\left(q^{N k}\right)_{\lambda}^{\left(q, q^{k}\right)}}{h_{\lambda}\left(q, q^{k}\right)} .
$$

It can be rewritten as follows:

$$
\begin{aligned}
& \left\langle P_{\lambda}\left(z ; q, q^{k}\right)\right\rangle \\
& =\frac{\left(q^{N k}\right)_{\lambda}^{\left(q, q^{k}\right)}}{h_{\lambda}\left(q, q^{k}\right)} \prod_{i=1}^{N} \frac{\Gamma_{q}\left(u_{1}+1+(N-j) k+\lambda_{i}\right) \Gamma_{q}\left(u_{1}+u_{2}+2+(2 N-j-1) k\right)}{\Gamma_{q}\left(u_{1}+1+(N-j) k\right) \Gamma_{q}\left(u_{1}+u_{2}+2+(2 N-j-1) k+\lambda_{i}\right)} \\
& =\frac{\left(q^{N k}\right)_{\lambda}^{\left(q, q^{k}\right)}\left(q^{u_{1}+1+(N-1) k}\right)_{\lambda}^{\left(q, q^{k}\right)}}{h_{\lambda}\left(q, q^{k}\right)\left(q^{u_{1}+u_{2}+2+2(N-1) k}\right)_{\lambda}^{\left(q, q^{k}\right)}} .
\end{aligned}
$$




\section{References}

[1] S. Lukyanov and Ya. Pugai, "Bosonization of ZF algebras: Direction toward deformed Virasoro algebra," J. Exp. Theor. Phys. 82, 1021-1045 (1996) [Zh. Eksp. Teor. Fiz. 109, 1900-1947 (1996)] [arXiv:hep-th/9412128].

[2] E. Frenkel and N. Reshetikhin, "Quantum Affine Algebras and Deformations of the Virasoso and $\mathcal{W}$-Algebras," Commun. Math. Phys. 178, 237-264 (1996) arXiv:q-alg/9505025].

[3] J. Shiraishi, H. Kubo, H. Awata and S. Odake, "A quantum deformation of the Virasoro algebra and the Macdonald symmetric functions," Lett. Math. Phys. 38, 33-51 (1996) arXiv:q-alg/9507034.

[4] B. Feigin and E. Frenkel, "Quantum $\mathcal{W}$-Algebras and Elliptic Algebras," Commun. Math. Phys. 178, 653-678 (1996) arXiv:q-alg/9508009].

[5] H. Awata, H. Kubo, S. Odake and J. Shiraishi, "Quantum $\mathcal{W}_{N}$ Algebras and Macdonald Polynomials," Commun. Math. Phys. 179, 401-416 (1996) arXiv:q-alg/9508011.

[6] K. Mimachi and Y. Yamada, "Singular vectors of the Virasoro Algebra in Terms of Jack Symmetric Polynomials," Commun. Math. Phys. 174, 447-455 (1995).

[7] I. G. Macdonald, Symmetric functions and Hall Polynomials, 2nd ed., Oxford University Press (1995).

[8] H. Awata, H. Kubo, Y. Morita, S. Odake and J. Shiraishi, "Vertex Operators of the $q$-Virasoro Algebra; Defining Relations, Adjoint Actions and Four Point Functions," Lett. Math. Phys. 41, 65-78 (1997) arXiv:q-alg/9604023.

[9] A. A. Kadeishvili, "Vertex operators for deformed Virasoro algebra," JETP Lett. 63, 917-923 (1996) [Pisma Zh. Eksp. Teor. Fiz. 63, 876-881 (1996)] arXiv:hep-th/9604153.

[10] M. Jimbo and J. Shiraishi, "A Coset-Type Construction for the Deformed Virasoro Algebra," Lett. Math. Phys. 43, 173-185 (1998) [arXiv:q-alg/9709037].

[11] L. F. Alday, D. Gaiotto and Y. Tachikawa, "Liouville Correlation Functions from Fourdimensional Gauge Theories," Lett. Math. Phys. 9, 167-197 (2010) [arXiv:0906.3219 [hep-th]].

[12] N. Wyllard, " $A_{N-1}$ conformal Toda field theory correlation functions from conformal $\mathcal{N}=2$ $S U(N)$ quiver gauge theories," JHEP 0911, 002 (2009) [arXiv:0907.2189 [hep-th]].

[13] A. Marshakov, A. Mironov and A. Morozov, "Combinatorial expansions of conformal blocks," Theor. Math. Phys. 164, 831-852 (2010) [Teor. Mat. Fiz. 164, 3-27 (2010)] arXiv:0907.3946 [hep-th]];

A. Mironov, S. Mironov, A. Morozov and And. Morozov, "CFT exercises for the needs of AGT," arXiv:0908.2064 [hep-th];

A. Mironov and A. Morozov, "The power of Nekrasov functions," Phys. Lett. B 680, 188-194 (2009) arXiv:0908.2190 [hep-th]]; 
A. Mironov and A. Morozov, "On AGT relation in the case of $U(3)$," Nucl. Phys. B 825, 1-37 (2010) arXiv:0908.2569 [hep-th]];

A. Marshakov, A. Mironov and A. Morozov, "On non-conformal limit of the AGT relations," Phys. Lett. B 682, 125-129 (2009) [arXiv:0909.2052 [hep-th]].

[14] R. Dijkgraaf and C. Vafa, "Toda Theories, Matrix Models, Topological Strings, and $N=2$ Gauge Systems," arXiv:0909.2453 [hep-th].

[15] A. Marshakov, A. Mironov and A. Morozov, "Zamolodchikov asymptotic formula and instanton expansion in $\mathcal{N}=2$ SUSY $N_{f}=2 N_{c}$ QCD," JHEP 0911, 048 (2009) arXiv:0909.3338 [hep-th]];

A. Mironov and A. Morozov, "Proving AGT relations in the large- $c$ limit," Phys. Lett. B 682, 118-124 (2009) [arXiv:0909.3531 [hep-th]];

A. Mironov and A. Morozov, "Nekrasov functions and exact Bohr-Zommerfeld integrals," JHEP 1004, 040 (2010) [arXiv:0910.5670 [hep-th]];

A. Mironov and A. Morozov, "Nekrasov functions from exact Bohr-Sommerfeld periods: the case of $S U(N), "$ J. Phys. A 43, 195401 (2010) [arXiv:0911.2396 [hep-th]].

[16] H. Itoyama, K. Maruyoshi and T. Oota, "The Quiver Matrix Model and 2d-4d Conformal Connection," Prog. Theor. Phys. 123, 957-987 (2010) [arXiv:0911.4244 [hep-th]].

[17] A. Mironov, A. Morozov and Sh. Shakirov, "Matrix model conjecture for exact BS periods and Nekrasov functions," JHEP 1002, 030 (2010) [arXiv:0911.5721 [hep-th]];

A. Mironov, A. Morozov and Sh. Shakirov, "Conformal blocks as Dotsenko-Fateev integral discriminants," Int. J. Mod. Phys. A 25, 3173-3207 (2010) [arXiv:1001.0563 [hep-th]];

A. Mironov, A. Morozov and And. Morozov, "Conformal blocks and generalized Selberg integrals," Nucl. Phys. B 843, 534-557 (2011) [arXiv:1003.5752 [hep-th]];

A. Mironov, A. Morozov and Sh. Shakirov, "Towards a proof of AGT conjecture by methods of matrix models," Int. J. Mod. Phys. A 27, 1230001 (2012) [arXiv:1011.5629 [hep-th]];

A. Mironov, A. Morozov and Sh. Shakirov, "A direct proof of AGT conjecture at $\beta=1$," JHEP 1102, 067 (2011) [arXiv:1012.3137 [hep-th]].

[18] V. A. Fateev and A. V. Litvinov, "On AGT conjecture," JHEP 1002, 014 (2010) arXiv:0912.0504 [hep-th]];

V. A. Fateev and A. V. Litvinov, "Integrable structure, W-symmetry and AGT relation," JHEP 1201, 051 (2012) [arXiv:1109.4042 [hep-th]].

[19] H. Itoyama and T. Oota, "Method of generating $q$-expansion coefficients for conformal block and $\mathcal{N}=2$ Nekrasov function by $\beta$-deformed matrix model," Nucl. Phys. B 838, 298-330 (2010) [arXiv:1003.2929 [hep-th]].

[20] H. Itoyama, T. Oota and N. Yonezawa, "Massive scaling limit of the $\beta$-deformed matrix model of Selberg type," Phys. Rev. D 82, 085031 (2010) arXiv:1008.1861 [hep-th]]. 
[21] V. A. Alba, V. A. Fateev, A. V. Litvinov and G. M. Tarnopolskiy, "On Combinatorial Expansion of the Conformal Blocks Arising from AGT Conjecture," Lett. Math. Phys. 98, 33-64 (2011) [arXiv:1012.1312 [hep-th]].

[22] N. Wyllard, "Coset conformal blocks and $\mathcal{N}=2$ gauge theories," arXiv:1109.4264 [hep-th].

[23] B. Estienne, V. Pasquier, R. Santachiara and D. Serban, "Conformal blocks in Virasoro and W theories: Duality and the Calogero-Sutherland model," Nucl. Phys. B 860, 377-420 (2012) arXiv:1110.1101 [hep-th]].

[24] H. Zhang and Y. Matsuo, "Selberg Integral and SU(N) AGT Conjecture," JHEP 1112, 106 (2011) [arXiv:1110.5255 [hep-th]].

[25] A. A. Belavin, M. A. Bershtein, B. L. Feigin, A. V. Litvinov and G. M. Tarnopolsky, "Instanton moduli spaces and bases in coset conformal field theory," Commun. Math. Phys. 319, 269-301 (2013) [arXiv:1111.2803 [hep-th]].

[26] S. Kanno, Y. Matsuo and H. Zhang, "Virasoro constraint for Nekrasov instanton partition function," JHEP 1210, 097 (2012) [arXiv:1207.5658 [hep-th]];

S. Kanno, Y. Matsuo and H. Zhang, "Extended Conformal Symmetry and Recursion Formulae for Nekrasov Partition Function," JHEP 1308, 028 (2013) [arXiv:1306.1523 [hep-th]].

[27] M.-C. Tan, "M-theoretic derivations of 4d-2d dualities: from a geometric Langlands duality for surfaces, to the AGT correspondence, to integrable systems," JHEP 1307, 171 (2013) arXiv:1301.1977 [hep-th]].

[28] A. Morozov and A. Smirnov, "Towards the Proof of AGT Relations with the Help of the Generalized Jack Polynomials," Lett. Math. Phys. 104 585-612 (2014) arXiv:1307.2576 [hepth]].

[29] S. Mironov, And. Morozov and Y. Zenkevich, "Generalized Jack polynomials and the AGT relations for the $S U(3)$ group," JETP Lett. 99, 109-113 (2014) [arXiv:1312.5732 [hep-th]].

[30] Y. Matsuo, C. Rim and H. Zhang, "Construction of Gaiotto states with fundamental multiplets through degenerate DAHA," JHEP 1409, 028 (2014) [arXiv:1405.3141 [hep-th]].

[31] H. Itoyama and R. Yoshioka, "Developments of theory of effective prepotential from extended Seiberg-Witten system and matrix models," Prog. Theor. Exp. Phys. 11B103 (2015) arXiv:1507.00260 [hep-th]].

[32] A. Nedelin and M. Zabzine, "q-Virasoro constraints in matrix models," arXiv:1511.03471 [hep-th].

[33] H. Awata and Y. Yamada, "Five-dimensional AGT conjecture and the deformed Virasoro algebra," JHEP 1001, 125 (2010) [arXiv:0910.4431 [hep-th]].

[34] H. Awata and Y. Yamada, "Five-Dimensional AGT Relation and the Deformed $\beta$-Ensemble," Prog. Theor. Phys. 124, 227-262 (2010) [arXiv:1004.5122 [hep-th]]. 
[35] A. Mironov, A. Morozov, Sh. Shakirov and A. Smirnov, "Proving AGT conjecture as HS duality: Extension to five dimensions," Nucl. Phys. B 855, 128-151 (2012) arXiv:1105.0948 [hep-th]].

[36] F. Nieri, S. Pasquetti and F. Passerini, "3d and 5d Gauge Theory Partition Functions as q-deformed CFT Correlators," Lett. Math. Phys. 105, 109-148 (2015) arXiv:1303.2626 [hepth]].

[37] M.-C. Tan, "An M-theoretic derivation of a 5d and 6d AGT correspondence, and relativistic and elliptized integrable systems," JHEP 1312, 031 (2013) [arXiv:1309.4775 [hep-th]].

[38] F. Nieri, S. Pasquetti, F. Passerini and A. Torrielli, "5D partition functions, q-Virasoro systems and integrable spin-chains" JHEP 1412, 040 (2014) [arXiv:1312.1294 [hep-th]].

[39] Y. Ohkubo, "Existence and orthogonality of generalized Jack polynomials and its $q$ deformation," arXiv:1404.5401 [math-ph].

[40] Y. Zenkevich, "Generalized Macdonald polynomials, spectral duality for conformal blocks and AGT correspondence in five dimensions," JHEP 1505, 131 (2015) [arXiv:1412.8592 [hep-th]].

[41] H. Awata, H. Fujino and Y. Ohkubo, "Crystallization of deformed Virasoro algebra, DingIohara-Miki algebra and 5D AGT correspondence," arXiv:1512.08016 [math-ph].

[42] T. Kimura and V. Pestun, "Quiver W-algebras," arXiv:1512.08533 [hep-th].

[43] A. Morozov and Y. Zenkevich, "Decomposing Nekrasov Decomposition," arXiv:1510.01896 [hep-th].

[44] A. Iqbal, C. Kozçaz and S.-T. Yau, "Elliptic Virasoro Conformal Blocks," arXiv:1511.00458 [hep-th].

[45] F. Nieri, "An elliptic Virasoro symmetry in 6d," arXiv:1511.00574 [hep-th].

[46] A. Mironov, A. Morozov and Y. Zenkevich, "On elementary proof of AGT relations from six dimensions," arXiv:1512.06701 [hep-th].

[47] J. Kaneko, " $q$-Selberg integrals and Macdonald polynomials," Ann. scient. Éc. Norm. Sup. 29, 583-637 (1996).

[48] V. Belavin and B. Feigin, "Super Liouville conformal blocks from $\mathcal{N}=2 S U(2)$ quiver gauge theories," JHEP 1107, 079 (2011) [arXiv:1105.5800 [hep-th]].

[49] T. Nishioka and Y. Tachikawa, "Central charges of para-Liouville and Toda theories from M-5-branes," Phys. Rev. D 84, 046009 (2011) [arXiv:1106.1172 [hep-th]].

[50] A. Belavin, V. Belavin and M. Bershtein, "Instantons and 2d Superconformal field theory," JHEP 1109, 117 (2011) [arXiv:1106.4001 [hep-th]]. 
[51] G. Bonelli, K. Maruyoshi and A. Tanzini, "Instantons on ALE spaces and super Liouville conformal field theories," JHEP 1108, 056 (2011) [arXiv:1106.2505 [hep-th]];

G. Bonelli, K. Maruyoshi and A. Tanzini, "Gauge Theories on ALE Space and Super Liouville Correlation Functions," Lett. Math. Phys. 101, 103-124 (2012) [arXiv:1107.4609 [hep-th]].

[52] Y. Ito, "Ramond sector of super Liouville theory from instantons on an ALE space," Nucl. Phys. B 861, 387-402 (2012) [arXiv:1110.2176 [hep-th]].

[53] M. N. Alfimov and G. M. Tarnopolsky, "Parafermionic Liouville field theory and instantons on ALE spaces," JHEP 1202, 036 (2012) arXiv:1110.5628 [hep-th]].

[54] V. Belavin and N. Wyllard, "N $=2$ superconformal blocks and instanton partition functions," JHEP 1206, 173 (2012) [arXiv:1205.3091 [hep-th]].

[55] A. Belavin and B. Mukhametzhanov, " $N=1$ superconformal blocks with Ramond fields from AGT correspondence," JHEP 1301, 178 (2013) [arXiv:1210.7454 [hep-th]].

[56] A. A. Belavin, M. A. Bershtein and G. M. Tarnopolsky, "Bases in coset conformal field theory from AGT correspondence and Macdonald polynomials at the roots of unity," arXiv:1211.2788 [hep-th].

[57] M. N. Alfimov, A. A. Belavin and G. M. Tarnopolsky, "Coset conformal field theory and instanton counting on $\mathbb{C}^{2} / \mathbb{Z}_{p}$, , JHEP 1308, 134 (2013) [arXiv:1306.3938 [hep-th]].

[58] H. Itoyama, T. Oota and R. Yoshioka, "2d-4d Connection between $q$-Virasoro/W Block at Root of Unity Limit and Instanton Partition Function on ALE Space," Nucl. Phys. B 877, 506-537 (2013) [arXiv:1308.2068 [hep-th]];

H. Itoyama, T. Oota and R. Yoshioka, "q-Virasoro algebra at root of unity limit and 2d-4d connection," J. Phys. Conf. Ser. 474, 012022 (2013);

H. Itoyama, T. Oota and R. Yoshioka, " $q$-Virasoro/W Algebra at Root of Unity and Parafermions," Nucl. Phys. B 889, 25-35 (2014) [arXiv:1408.4216 [hep-th]].

[59] L. Spodyneiko, "AGT correspondence: Ding-Iohara algebra at roots of unity and LepowskyWilson construction," J. Phys. A 48, 275404 (2015) [arXiv:1409.3465 [hep-th]].

[60] R. Yoshioka, "The integral representation of solutions of KZ equation and a modification by $\mathcal{K}$ operator insertion," arXiv:1512.01084 [hep-th].

[61] N. A. Nekrasov, "Seiberg-Witten Prepotential From Instanton Counting," Adv. Theor. Math. Phys. 7, 831-864 (2004) arXiv:hep-th/0206161.

[62] N. Nekrasov and A. Okounkov, "Seiberg-Witten Theory and Random Partitions," in The Unity of Mathematics, in Honor of the Ninetieth Birthday of I.M. Gelfand, Progress of Mathematics Vol. 244, 525-596, ed. by P. Etingof, V. Retakh and I.M. Singer, Birkh" auser, Boston (2006) arXiv:hep-th/0306238.

[63] H. Nakajima and K. Yoshioka, "Instanton counting on blowup. II. $K$-theoretic partition function," Transfor. Groups 10, 489-519 (2005) arXiv:math/0505553 [math.AG]]. 
[64] K. Aomoto, "On Elliptic Product Formulas for Jackson Integrals Associated with Reduced Root Systems," J. Alg. Geom. 8, 115-126 (1998).

[65] R. Askey, "Some Basic Hypergeometric Extensions of Integrals of Selberg and Andrews," SIAM J. Math. Anal. 11, 938-951 (1980).

[66] L. Habsieger, "Une q-Intégrale de Selberg et Askey," SIAM J. Math. Anal. 19, 1475-1489 (1988).

[67] K. W. J. Kadell, "A Proof of Askey's Conjectured q-Analogue of Selberg's Integral and a Conjecture of Morris," SIAM J. Math. Anal. 19, 969-986 (1988). 